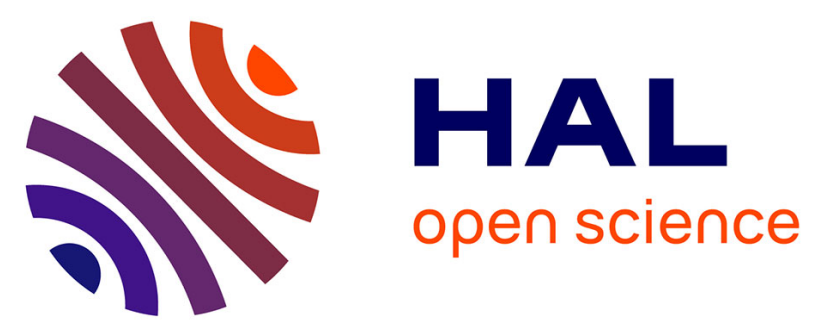

\title{
Numerical and Experimental Study of First Symmetric and Antisymmetric Lamb Wave Modes Generated and Received by Dual-PZTs in a Composite Plate
}

\author{
Emmanuel Lizé, Charles Hudin, Marc Rebillat, Nazih Mechbal, Christian
} Bolzmacher

\section{To cite this version:}

Emmanuel Lizé, Charles Hudin, Marc Rebillat, Nazih Mechbal, Christian Bolzmacher. Numerical and Experimental Study of First Symmetric and Antisymmetric Lamb Wave Modes Generated and Received by Dual-PZTs in a Composite Plate. Structural Health Monitoring 2017, Sep 2017, Stanford, United States. 10.12783/shm2017/14053 . hal-01775214

\author{
HAL Id: hal-01775214 \\ https://hal.science/hal-01775214
}

Submitted on 17 May 2018

HAL is a multi-disciplinary open access archive for the deposit and dissemination of scientific research documents, whether they are published or not. The documents may come from teaching and research institutions in France or abroad, or from public or private research centers.
L'archive ouverte pluridisciplinaire HAL, est destinée au dépôt et à la diffusion de documents scientifiques de niveau recherche, publiés ou non, émanant des établissements d'enseignement et de recherche français ou étrangers, des laboratoires publics ou privés. 


\title{
Numerical and experimental study of first symmetric and antisymmetric Lamb wave modes generated and received by dual-PZTs in a composite plate
}

\author{
Emmanuel LIZÉ ${ }^{1}$, Charles HUDIN ${ }^{1}$, Marc RÉBILLAT ${ }^{2}$, Nazih MECHBAL ${ }^{2}$, \\ Christian BOLZMACHER ${ }^{1}$ \\ 1 CEA, LIST, Sensorial and Ambient Interfaces Laboratory, 91191 - Gif-sur-Yvette \\ CEDEX, France \\ 2 Processes and Engineering in Mechanics and Materials Laboratory \\ (PIMM, UMR CNRS 8006, Arts et Métiers ParisTech (ENSAM)), \\ 151, Boulevard de l'Hôpital, Paris, F-75013, France, \\ emmanuel.lize@cea.fr
}

\begin{abstract}
Structural Health Monitoring is a multidisciplinary field that provides processes whose aim is to monitor damages within structures. Most damage indexes are calculated using Lamb wave signals generated and received by surface-mounted piezoceramic transducers (PZT). The symmetric and antisymmetric Lamb wave modes have different velocities and tend to highlight different kinds of damages. In most SHM applications, a narrowband tone burst with a single frequency component is used as excitation signal. Using two dual PZTs (concentric disc and ring) allows one to tune the emitted signal properly in order to favor antisymmetric or symmetric mode propagation, and to decompose both mode contributions in the received signal. Hence those easy-to-settle devices enable one to detect small damages with appropriate frequencies while guarantying optimal energy efficiency in emission and reception. In this article, a simple model based on the Kirchhoff-Love beam theory is used to estimate the amplitude of the $\mathrm{S}_{0}$ and $\mathrm{A}_{0}$ waves generated by a dual PZT mounted on a composite plate. This estimation is confronted to experimental measurements obtained with a laser Doppler vibrometer (for the $\mathrm{A}_{0}$ mode only). Due to the limited prediction capabilities of the analytical model, a numerical model based on finite element simulations (FEM) in SDTools is introduced. First results indicate good agreement between simulation and measurements. In future studies, the best sensor/actuator combination, the maximum distance between transducers as well as the frequencies to be used for pitch-catch and pulse-echo configurations in a context of delamination detection for SHM application will be determined.
\end{abstract}

\section{INTRODUCTION}

The field of Structural Health Monitoring (SHM) has been continuously evolving for the last 20 years. The introduction of new technologies and improvements in 
computer science lead to great innovations on existing techniques, such as signal processing, allowing the creation of more complex methods and leading to more accurate results in SHM.

Damage detection using guided waves is one of the most used methods in SHM. Among all the methods employed to trigger and catch guided waves, the use of piezoelectric transducer (PZT) is one of the cheapest and easiest-to-settle. The usual method is based on the comparison of signals recorded on a "pristine" structure - the baseline - against signals obtained on a damaged structure. However, the resulting signal strongly depends on the structure's mechanical and geometrical properties, which are significantly altered in presence of damage or varying environmental conditions. This can lead to false detection when compared with the baseline data. Some solutions have been proposed using temperature compensation methods or extended baseline data, but in general "baseline-free" methods have to be achieved for efficient monitoring. Baseline-free is an abusive word (axiom II from [1]) since data is always needed to tell whether a structure is damaged or not (geometrical or electromechanical properties, threshold values, ...).

Among the existing baseline-free methods, one is based on the use of dual PZT. Those PZTs are composed of a concentric ring and disc (see Figure 2). Both parts can be used as actuator and sensor bringing more signal combinations than conventional PZT disc configurations, and allow the isolation of the $\mathrm{A}_{0}$ or $\mathrm{S}_{0}$ mode in the received signal [2]-[6]. The theoretical aspect justifying the use of those PZTs is well developed in [7]. A damage is detected using the damage-introduced mode conversion of the propagating waves [8].For example, a delamination in a composite structure attenuates the antisymmetric mode and reflects the symmetric one. Dual PZTs have been tested on aluminium [9] and composite [10] structures and are efficient even under variable environmental conditions [10]. The techniques involved require to know the amplitude ratio of the $\mathrm{A}_{0}$ wave against $\mathrm{S}_{0}$ wave.

The first part of this article describes a simple model based on Kirchhoff-Loves beam theory to estimate the wave amplitude over frequency on a composite plate. It has already been used in order to estimate the radiation of a PZT (flexural waves) on a glass plate [11]. The second part presents results obtained with the Finite Element Method (FEM) software SDTools. It consists of a simulation of the propagation of narrowband tone bursts triggered and measured using dual PZT mounted on a composite plate.

\section{SIMPLE MODEL BASED ON THE KIRCHHOFF-LOVE BEAM THEORY}

In SHM applications, PZT patches are mounted on thin structures in order to generate and measure Lamb waves. A perfectly glued thin transducer triggers a punctual radial force at its outer edge on the surface of the structure. In the neutral plane of the structure, this is equivalent to compression forces and bending moments that respectively activate longitudinal compression and transversal flexural waves. Depending on the structure's mechanical properties, the dimensions of the transducer and the frequency of the signal used for excitation, these waves excite one or several modes. They can be either symmetric or antisymmetric, with different velocities and amplitudes. This simple model allow the amplitude estimation of the first symmetric $\left(\mathrm{S}_{0}\right)$ and antisymmetric $\left(\mathrm{A}_{0}\right)$ modes triggered by a PZT glued on a plate with a simple beam model. This approach follows the development proposed in [12] which is actually 
an extension of the Classical Laminated Plate Theory. Some assumption are made: the beam is thin (thickness is really small compared to other dimensions); edge effects are neglected; different layers of the structure are perfectly glued to each other; the Elastic moduli of the different components have same orders of magnitude; the transversal shear is neglected and deformations in the y direction are not considered.

The displacement $U$ at any position $M(x, z)$ is equal to:

$$
\vec{U}(x, z, t)=\left(u(x, t)-z \frac{\partial w}{\partial x}(x, t)\right) \vec{x}+w(x, t) \vec{z}
$$

with $u$ and $w$ the longitudinal and transverse displacements of the neutral axis of the beam along $x$. It is considered that the constraints in the $x$ direction are predominant $\left(\sigma_{y y}=\sigma_{z z}=0\right)$ and the electrical potential of the PZT varies linearly with $z . \psi$ being the electrostatic potential, the electric field is $E_{z}=-z . \partial \psi / \partial z$.

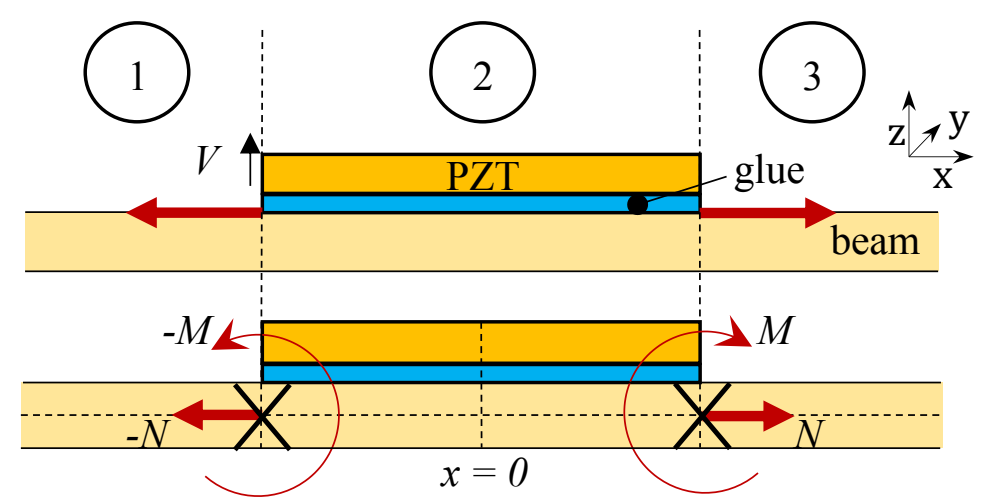

Figure 1. Pin force model of a PZT glued to a beam.

With these assumptions, [12] shows that the strain field $\sigma_{x x}$ and the electric displacement field $D_{z}$ of the $k$-th PZT layer can be written as:

$$
\left\{\begin{array}{l}
\sigma_{x x}=c_{k} \varepsilon_{x x}-e_{k} E_{z} \\
D_{z}=e_{k} \varepsilon_{x x}+\epsilon_{k} E_{z}
\end{array} \quad \text { with } \quad \varepsilon_{x x}=\frac{\partial u}{\partial x}-z \frac{\partial^{2} w}{\partial x^{2}}\right.
$$

where $\varepsilon_{x x}$ is the deformation field, $c_{k}$ the elastic compliance at constant electric field, $e_{k}$ the converse piezoelectric effect constant, and $\epsilon_{k}$ the permittivity at constant stress field (dielectric constants), for the $k$-th PZT layer.

The compression force $N$ and the bending moment $M$ are defined by:

$$
\left\{\begin{array}{c}
N=\int_{S} \sigma_{x x} d S=b \int_{z_{0}}^{z_{P}} \sigma_{x x}(x, z, t) d z \\
M=\int_{S} z \sigma_{x x} d S=b \int_{z_{0}}^{z_{P}} z \sigma_{x x}(x, z, t) d z
\end{array}\right.
$$

where $S=[-b / 2 ; b / 2] \times\left[z_{0} ; z_{P}\right]$ is the the section of the beam at abscissa $x$. The bending moment $M$ is a moment around the axis $(P, y)$, with $P$ the point with coordinates $(O, x)$. The voltage of the $k$-th PZT layer is $V_{k}=-\int_{z_{k-1}}^{z_{k}} E_{z} d z$.

With the K layers of the system, Eq.(2) leads to: 


$$
\begin{aligned}
& \begin{cases}N=A \frac{\partial u}{\partial x}-B \frac{\partial^{2} w}{\partial x^{2}}+\sum_{k \in[1 . . K]} \Xi_{k} V_{k} & A=\sum_{k=1}^{K} b h_{k} c_{k}, \\
M=B \frac{\partial u}{\partial x}-D \frac{\partial^{2} w}{\partial x^{2}}+\sum_{k \in[1 . . K]} \Theta_{k} V_{k} & \text { with } \quad B=\sum_{k=1}^{K} I_{1 k} c_{k}, \\
D & =\sum_{k=1}^{K} I_{2 k} c_{k},\end{cases} \\
& \text { and } \quad \Xi_{\mathrm{k}}=b e_{k}, \quad \Theta_{k}=\frac{I_{1 k}}{h_{k}} e_{k}=b \frac{z_{k}+z_{k-1}}{2} e_{k}, \\
& I_{1 k}=\int_{S} z d S=b \frac{z_{k}^{2}-z_{k-1}^{2}}{2}, \quad I_{2 k}=\int_{S} z^{2} d S=b \frac{z_{k}^{3}-z_{k-1}^{3}}{3}
\end{aligned}
$$

with $h_{k}=z_{k}-z_{k-1}, I_{1 k}$, and $I_{2 k}$ the thickness, linear moment and quadratic moment of the $k$-th layer, respectively. The equations of equilibrium give the following relations with $m$ being the mass per unit length of the structure if no other force is applied to the structure:

$$
\left\{\begin{array}{l}
-\frac{\partial^{2} M}{\partial x^{2}}+m \frac{\partial^{2} w}{\partial t^{2}}=0 \\
-\frac{\partial N}{\partial x}+m \frac{\partial^{2} u}{\partial t^{2}}=0
\end{array} \quad \text { with } m(x)=b \sum_{k=1}^{K} \rho_{k} h_{k}\right.
$$

Expressions of $M$ and $N$ in Eq.(4) are replaced in Eq.(5), for each portion $i \in$ $\{1,2,3\}$ of the structure (see Figure 1):

$$
\left\{\begin{array}{l}
-B_{i} \frac{\partial^{3} u_{i}}{\partial x^{3}}+D_{i} \frac{\partial^{4} w_{i}}{\partial x^{4}}+m_{i} \frac{\partial^{2} w_{i}}{\partial t^{2}}=0 \\
-A_{i} \frac{\partial^{2} u_{i}}{\partial^{2} x}+D_{i} \frac{\partial^{3} w_{i}}{\partial x^{3}}+m_{i} \frac{\partial^{2} u_{i}}{\partial t^{2}}=0
\end{array}\right.
$$

These equations allow propagative and evanescent solutions for the flexural waves $w$ and a propagative solution for compression waves $u$.

In this case study, the solution for each portion is given by:

$$
\begin{aligned}
& w_{1}(x)=T_{P 1}^{-} e^{i k_{1} x}+T_{E 1}^{-} e^{k_{1} x} \\
& w_{2}(x)=T_{P 2}^{-} e^{i k_{2} x}+T_{E 2}^{-} e^{k_{2} x}+T_{P 2}^{+} e^{-i k_{2} x}+T_{E 2}^{+} e^{-k_{2} x} \\
& w_{3}(x)=T_{P 3}^{+} e^{-i k_{3} x}+T_{E 3}^{+} e^{-k_{3} x} \\
& u_{1}(x)=L_{P 1}^{-} e^{i q_{1} x} \\
& u_{2}(x)=L_{P 2}^{-} e^{i q_{2} x}+L_{P 2}^{+} e^{-i q_{2} x} \\
& u_{3}(x)=L_{P 3}^{+} e^{-i q_{3} x}
\end{aligned}
$$

$T_{P}$ and $T_{E}$ denote the complex amplitude of the propagative and evanescent wave of the transversal waves, $L_{P}$ denote the complex amplitude of the propagative wave of the longitudinal wave, whereas . ${ }^{+}$and.$^{-}$exponents represent the positive and negative directions of propagation along $x$ axis. The amplitude reduction through geometric spreading is not implemented but it could be included as proposed in [13] by considering that it is proportional to $1 / \sqrt{x}$. The wave numbers $k_{i}$ and $q_{i}$ are related to the pulsation $\omega$ by:

$$
\omega=\sqrt{\frac{D_{i}}{m_{i}}} k_{i}^{2}=\sqrt{\frac{A_{i}}{m_{i}}} q_{i}
$$


This method is applied on a $\left[0^{\circ}, 90^{\circ}\right]_{16}$ composite plate. In order to include the variations of mechanical properties of the composite plate according to the direction of the plies, 16 layers are considered and each sublayer has a Young's modulus related to the ply orientation. Results displayed in Figure $3 \mathrm{a}$ are obtained for waves propagating in a $0^{\circ}$ direction.

The bending moment and the compression force depends on the properties and dimensions of the PZT and the structure. They are proportional to the applied voltage: $M=\Theta_{p} V$ and $N=\Xi_{\mathrm{p}} V$.

Solutions given in Eq.(7) provide 12 unknown variables. At the junctions of the beam portion ( $x= \pm R$, with $R$ the radius of the PZT), the continuity of displacements and slopes as well as the equilibrium of bending moments and shear forces provide the 12 following equations needed to solve the problem:

$$
\left\{\begin{array} { c } 
{ w _ { 1 } ( - R ) = w _ { 2 } ( - R ) } \\
{ u _ { 1 } ( - R ) = u _ { 2 } ( - R ) } \\
{ w _ { 1 } ^ { \prime } ( - R ) = w _ { 2 } ^ { \prime } ( - R ) } \\
{ - D _ { 1 } w _ { 1 } ^ { \prime \prime } ( - R ) = - D _ { 2 } w _ { 2 } ^ { \prime \prime } ( - R ) \ldots } \\
{ \quad + B _ { 2 } u _ { 2 } ^ { \prime } ( - R ) + \Theta _ { p } V } \\
{ A _ { 1 } u _ { 1 } ^ { \prime } ( - R ) = A _ { 2 } u _ { 2 } ^ { \prime } ( - R ) \ldots } \\
{ \quad + B _ { 2 } w _ { 2 } ^ { \prime \prime } ( - R ) + \Xi _ { p } V } \\
{ D _ { 1 } w _ { 1 } ^ { \prime \prime \prime } ( - R ) = D _ { 2 } w _ { 2 } ^ { \prime \prime \prime } ( - R ) }
\end{array} \quad \left\{\begin{array}{c}
w_{2}(R)=w_{3}(R) \\
u_{2}(R)=u_{3}(R) \\
w_{2}{ }^{\prime}(R)=w_{3}{ }^{\prime}(R) \\
-D_{2} w_{2}^{\prime \prime}(R)=-D_{3} w_{3}^{\prime \prime}(R) \ldots \\
\quad+B_{3} u_{3}^{\prime}(R)-\Theta_{p} V \\
A_{2} u_{2}^{\prime}(R)=A_{3} u_{3}^{\prime}(R) \ldots \\
\quad+B_{3} w_{3}^{\prime \prime}(R)-\Xi_{p} V \\
D_{2} w_{2}^{\prime \prime \prime}(R)=D_{3} w_{3}^{\prime \prime \prime}(R)
\end{array}\right.\right.
$$

The proposed model is extended to dual PZTs, which are composed of a concentric ring and disc as shown in Figure 2. The disc behavior is estimated using the model with a PZT of diameter $\mathrm{d} 3$. The ring model is obtained by subtracting the model obtained with a PZT of diameter $\mathrm{d} 2$ to the model obtained with a PZT of diameter $\mathrm{d} 1$. The dual PZT model is the sum of the model computed for the disc and the one computed for the ring.

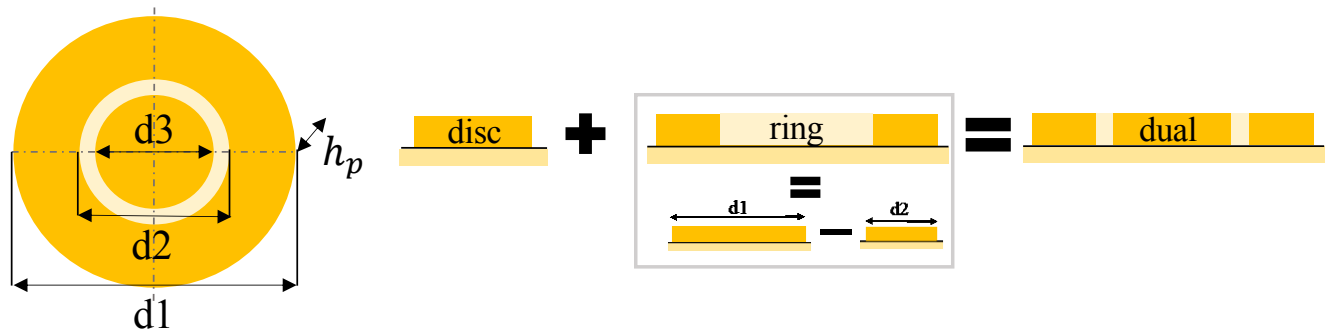

Figure 2. Theoretical representation of a dual PZT.

\section{COMPARISON WITH EXPERIMENTAL RESULTS}

Two dual PZTs with dimensions $\mathrm{d} 1=30 \mathrm{~mm}, \mathrm{~d} 2=10.5 \mathrm{~mm}, \mathrm{~d} 3=$ $9.5 \mathrm{~mm}, h_{p}=0.5 \mathrm{~mm}$ are mounted on a $\left[0^{\circ}, 90^{\circ}\right]_{16}$ composite plate of $910 \times$ $650 \times 2 \mathrm{~mm}^{3}$. The PZTs are glued with a ultrasonic testing couplant and placed as far as possible from the edges in order to minimize wave reflections in measurements. The PZT are separated by $250 \mathrm{~mm}$. 
TABLE I. ELECTRO-MECHANICAL PROPERTIES OF THE PZT, GLUE AND PLATE

\begin{tabular}{|c|c|c|c|c|c|c|}
\hline & $\rho\left[\mathrm{kg} \cdot \mathrm{m}^{-3}\right]$ & $h[\mathrm{~mm}]$ & $Y[G P a]$ & $c\left[m^{2} / N\right]$ & $e\left[C . m^{-2}\right]$ & $\epsilon$ \\
\hline PZT & 7850 & 0.5 & 59 & $-208.10^{-12}$ & -6.06 & 1900 \\
\hline glue & 1100 & $1.10^{-6}$ & 5.9 & & & \\
\hline plate & 1554 & 2 & $140^{0^{\circ}} \sim 9^{90^{\circ}}$ & & & \\
\hline
\end{tabular}

In order to evaluate the amplitude of the $\mathrm{A}_{0}$ waves, a sweep signal from 0 to $250 \mathrm{kHz}$ is triggered with different parts of the dual PZT and the velocity of the plate is measured by a laser Doppler vibrometer pointing $100 \mathrm{~mm}$ away from the center of the actuator. The transfer function $H$ is computed by dividing the Fast Fourier Transform (FFT) of the received signal by the FFT of the emitted signal (see Figure 3b.).
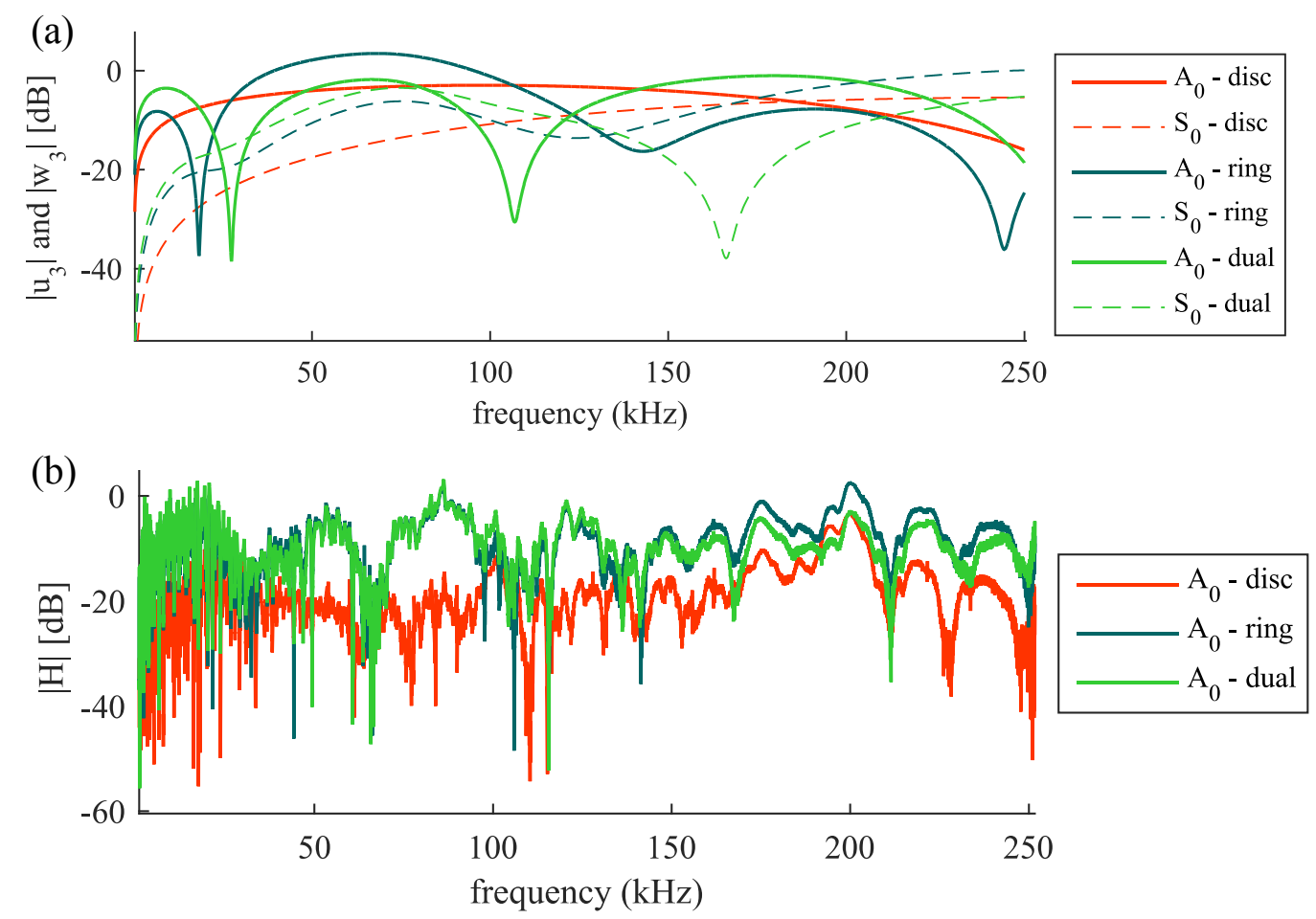

Figure 3. (a) Theoretical estimation of the relative amplitude between $\mathrm{A}_{0}$ and $\mathrm{S}_{0}$ waves obtained at $100 \mathrm{~mm}$ from a dual PZT; (b) Transfer function obtained with a sweep with different part of the dual PZT and a laser vibrometer at $100 \mathrm{~mm}$ from the PZT.

Theoretical results of Figure 3a show the interest of this approach. It can be seen form these curves that for example a signal triggered @148 kHz with the disc will radiate more $A_{0}$ mode than the ring. We can also see that with a signal triggered by the dual PZT (both parts at the same time), the $\mathrm{A}_{0}$ mode @110 kHz is $20 \mathrm{~dB}$ lower than the $\mathrm{S}_{0}$ mode whereas $\mathrm{A}_{0}$ mode @ $170 \mathrm{kHz}$ is $40 \mathrm{~dB}$ higher than the $\mathrm{S}_{0}$ mode.

Figure $3 \mathrm{~b}$ shows the experimental results obtained for a disc, a ring and a dual PZT for the A0 mode. Even if these graphs (Figure 3) show a certain tendency between theory and experiments, clear identification of the desired excitation frequencies for mode selection is challenging. First, it can be noticed that a wave triggered by the disc is weaker than one produced by the ring, and that makes sense since the surface of the 
ring part of the PZT is about two times the area of the disc. Moreover, the experimental response also contains modes attributable to the geometrical properties of the structure that are not taken into account in the theoretical model and may hide the non-radiating modes. A composite plate is a complex structure and confronted to experimental measurements, the simple beam theory model seems not to be appropriate to predict the amplitude of the two Lamb modes. This is the reason why the authors have decided to use a FEM software for further investigations.

\section{FEM SIMULATION WITH SDTOOLS}

The same configuration of the plate with two dual PZTs with electromechanical properties from TABLE I is implemented in SDTools. The used signal is a narrowband tone burst (a sine filtered by a Hanning window) with 5 cycles. It is triggered with a central frequency from 50 to $170 \mathrm{kHz}$ by steps of $5 \mathrm{kHz}$ and compared to experimental values (Figure $4 \mathrm{a})$.

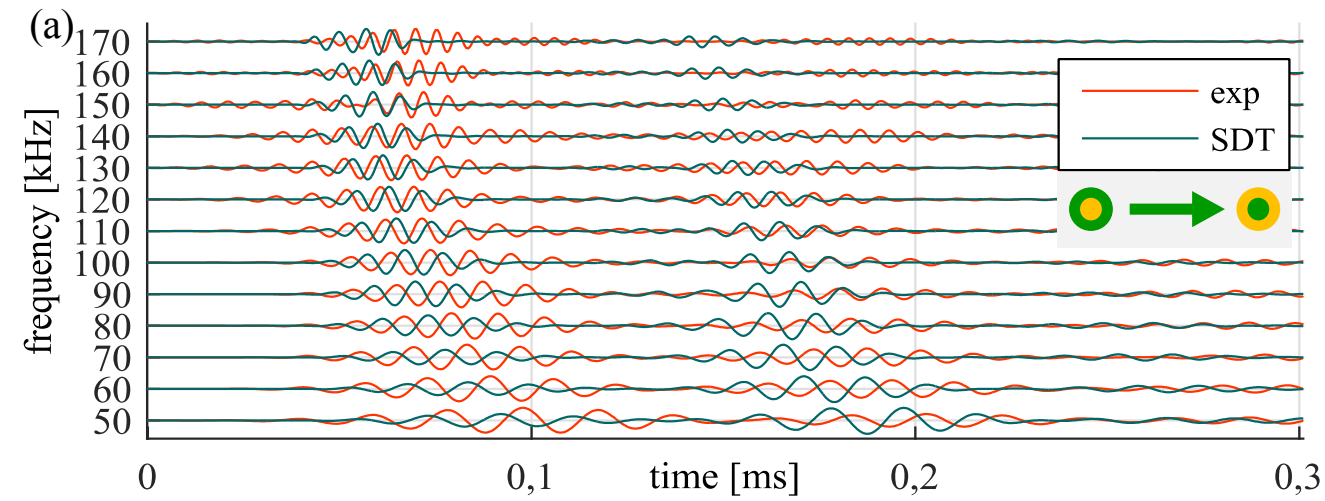

(b)

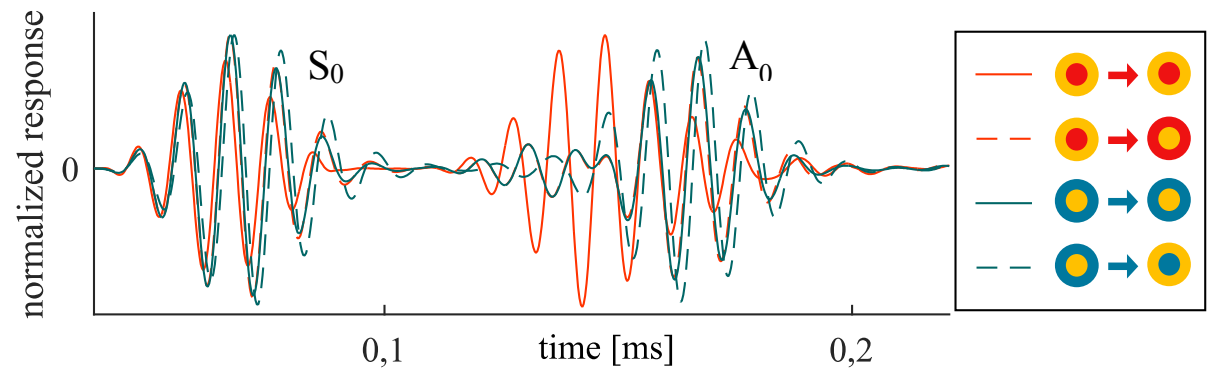

Figure 4. Normalized response obtained with a 5 cycle narrowband signal excitation with two dual PZTs mounted on a composite plate; (a) Comparison between FEM simulation and experimental results with increasing frequencies (combination ring - disc); (b) Comparison between different actuator-sensor combinations obtained via FEM simulation in SDTools.

Figure 4a shows $\mathrm{A}_{0}$ and $\mathrm{S}_{0}$ waves received by the disc of a dual PZT placed $250 \mathrm{~mm}$ away from the ring used as actuator. Experimental signals are post-treated with a Daubechies wavelet. Time of Arrival of SDTools simulation fits well experimental results (especially for frequencies between 110 and $140 \mathrm{kHz}$ ) and in both cases the ratio of amplitude of the $\mathrm{S}_{0}$ mode against $\mathrm{A}_{0}$ mode tends to increase with the frequency. Same observations (not displayed in this paper) have been observed by the authors for measurements obtained with other actuator-sensor combinations. 
These first results show promising perspectives to study the potential of dual PZT with FEM simulation. For example, Figure $4 \mathrm{~b}$ points out that received $\mathrm{S}_{0}$ waves tend to be really similar for all transducer combinations whereas the $A_{0}$ wave dispersion and time of arrival is more combination-dependent. Mode decomposition can then be applied by adding or subtracting signals.

\section{CONCLUSION}

This article presents a simple model based on the Kirchhoff-Love beam theory in order to compute the amplitude of the $A_{0}$ and $S_{0}$ waves propagating in a composite structure, triggered by dual PZTs. After a comparison with experimental values, this model does not seem to be adapted for proper estimation in the case of composite materials and shows the need for a more complex model. The first results obtained with a FEM simulation in SDTools with narrowband signals have displayed some interesting and realistic results. The authors will pursue investigations on dual PZTs by testing different scenarios and experiment the detection and localization of damages with baseline-free techniques in improved FEM simulations.

\section{REFERENCES}

1. K. Worden, C. R. Farrar, G. Manson, and G. Park, "The fundamental axioms of structural health monitoring," Proc. R. Soc. Math. Phys. Eng. Sci., vol. 463, no. 2082, pp. 1639-1664, Jun. 2007.

2. H. Sohn, H. J. Lim, C. M. Yeum, and J.-B. Ihn, Reference free inconsistency detection system. Google Patents, 2015.

3. Y.-K. An and H. Sohn, "Instantaneous crack detection under varying temperature and static loading conditions," Struct. Control Health Monit., vol. 17, no. 7, pp. 730-741, Nov. 2010.

4. C. M. Yeum, H. Sohn, and J. B. Ihn, "Delamination detection in a composite plate using a dual piezoelectric transducer network," 2011, p. 798406.

5. C. M. Yeum, H. Sohn, J. B. Ihn, and H. J. Lim, "Instantaneous delamination detection in a composite plate using a dual piezoelectric transducer network," Compos. Struct., vol. 94, no. 12, pp. 3490-3499, Dec. 2012.

6. C. M. Yeum, H. Sohn, H. J. Lim, and J. B. Ihn, "Reference-free delamination detection using Lamb waves: REFERENCE-FREE DELAMINATION DETECTION," Struct. Control Health Monit, Aug. 2013.

7. C. M. Yeum, H. Sohn, and J. B. Ihn, "Lamb wave mode decomposition using concentric ring and circular piezoelectric transducers," Wave Motion, vol. 48, no. 4, pp. 358-370, Jun. 2011.

8. Z. Su and L. Ye, Identification of damage using Lamb waves: from fundamentals to applications. Berlin: Springer-Verlag, 2009.

9. H. Sohn and S. B. Kim, "Development of dual PZT transducers for reference-free crack detection in thin plate structures," IEEE Trans. Ultrason. Ferroelectr. Freq. Control, vol. 57, no. 1, pp. 229240, Jan. 2010.

10. H. J. Lim, H. Sohn, C. M. Yeum, and J. M. Kim, "Reference-free damage detection, localization, and quantification in composites," J. Acoust. Soc. Am., vol. 133, no. 6, pp. 3838-3845, Jun. 2013.

11. C. Hudin, "Local friction modulation using non-radiating ultrasonic vibrations," Proceedings of World Haptics Conference 2017, 2017.

12. J. Ducarne, "Modélisation et optimisation de dispositifs non-linéaires d'amortissement de structures par systèmes piézoélectriques commutés," Paris, CNAM, 2009.

13. C. Heinze, S. Duczek, M. Sinapius, and P. Wierach, "A minimal model-based approach for the fast approximation of wave propagation in complex structures," Struct. Health Monit., p. 1475921717697509 , Mar. 2017.

14. V. Giurgiutiu, Structural health monitoring with piezoelectric wafer active sensors. Amsterdam: Academic Press/Elsevier, 2008. 